\title{
Persistent currents in mesoscopic rings and conformal invariance
}

\author{
Malte Henkel and Dragi Karevski \\ Laboratoire de Physique des Matériaux, [] \\ Université Henri Poincaré Nancy I, B.P. 239, \\ F - 54506 Vandœuvre-lès-Nancy Cedex, France
}

\begin{abstract}
The effect of point defects on persistent currents in mesoscopic rings is studied in a simple tight-binding model. Using an analogy with the treatment of the critical quantum Ising chain with defects, conformal invariance techniques are employed to relate the persistent current amplitude to the Hamiltonian spectrum just above the Fermi energy. From this, the dependence of the current on the magnetic flux is found exactly for a ring with one or two point defects.

The effect of an aperiodic modulation of the ring, generated through a binary substitution sequence, on the persistent current is also studied. The flux-dependence of the current is found to vary remarkably between the Fibonacci and the Thue-Morse sequences.
\end{abstract}

PACS: 05.20.-y, 64.60.-i, 73.23.-b

Keywords: conformal invariance, quantum chains, defects, mesoscopic systems, persistent currents

\footnotetext{
${ }^{1}$ Dedicated to Prof. J. Zittartz on the occasion of his $60^{\text {th }}$ birthday.

${ }^{2}$ Unité Mixte de Recherche CNRS No. 7556.
} 
The study of the critical behaviour of spin systems close to surfaces and interfaces has revealed a very rich phenomenology and has provided much insight into new phenomena. Two-dimensional systems have been of particular theoretical interest, because of fruitful connections with conformal invariance techniques, see [1, 2, 3, 4] for an introduction and review. Here, we present a new application of some conformal invariance methods to a simple problem of persistent currents, which we now specify.

Consider a small ring, made from a semiconducting or a normally conducting material. If the ring is threaded by a magnetic flux, one observes experimentally a persistent current, provided the ring has mesoscopic dimensions, that is, its diameter is in the nanometer range. Measured examples include $\mathrm{Au}$ [5] or GaAS-AlGaAs [6] rings. For larger rings, the current decreases and is no longer perceptible for rings of macroscopic size. The problem has received much attention. Non-interacting rings were studied in the classic papers by Kulik [0] and Büttiker et al. [8]. Disordered rings were considered in [9]. On the other hand, persistent currents for interacting $1 D$ electrons were first investigated in [10, 11]. See also [12, 13, 14, 15, 16, 17, 18, 19] and references therein. We refer to [20] for a review and to the nice book by Imry [21] for a detailed presentation of the physical background.

Here, we shall limit ourselves to a simple toy model which describes noninteracting (spin-less) electrons moving around a small, thin ring penetrated by an Aharonov-Bohm flux $\Phi$, see Figure 1. The flux $\Phi=\oint A d x=A L$, where $A$ is the vector potential and $L$ is the length of the ring. Assume that the ring is discretized into $N$ cells of size $a$. We use a simple tight-binding 


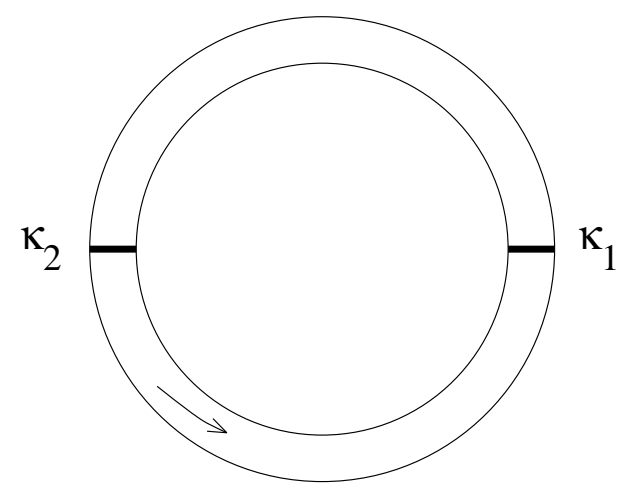

Figure 1: Aharonov-Bohm geometry. The places of the defects and their couplings $\kappa_{1,2}$ are indicated. The magnetic field is perpendicular to the plane.

model which describes the hopping of electrons from one cell to the next

$$
H=-t_{\text {hop }} \sum_{n=1}^{N}\left[\exp \left(2 \pi i \frac{\Phi}{\Phi_{0}} \frac{a}{L}\right) \psi^{\dagger}(n+1) \psi(n)+\text { h.c. }\right]
$$

where $t_{\text {hop }}$ is the hopping rate and $\Phi_{0}=h c / e$ is the flux quantum. Hamiltonians of this kind have been proposed earlier, see [11, [19], to study the effect of an Aharonov-Bohm flux on a Luttinger liquid. Our model (1) is, with respect to [11, 19], simplified even further in neglecting the charge-density interactions and only considering the zero-temperature case. On the other hand, we allow for point-like defects to occur in the ring, which phenomenologically lead to a local modification of the hopping rate, as already studied in [12.

We shall limit ourselves throughout to the case of half-filling, when exactly $M=N / 2$ particles are present and assume that $N$ is even. The flux $\Phi$ can be related to the phase

$$
\phi=2 \pi \Phi / \Phi_{0}
$$


of the wave function through the gauge transformation

$$
\psi(n) \rightarrow \exp \left(i \phi \frac{a}{L} n\right) \psi(n)
$$

leading to the conventional tight-binding Hamiltonian

$$
H=-t_{\text {hop }} \sum_{n=1}^{N}\left[\psi^{\dagger}(n+1) \psi(n)+\psi^{\dagger}(n) \psi(n+1)\right]
$$

with the boundary condition $\psi(N+1)=e^{-i \phi} \psi(1)$ and where $N$ is the number of sites. This is nothing but the classical Byers-Yang-Bloch result that the system is periodic in the flux $\Phi$ with period $\Phi_{0}$, see [11, 19, 20, 21 and references therein. In particular, the amplitude $j$ of the persistent current is obtained from the ground state energy $E_{0}(\Phi)$ of $H$ as

$$
j=-\frac{\partial E_{0}}{\partial \Phi}
$$

It is well known that for a perfect ring, $j=\left(t_{\text {hop }} / \pi\right)(\phi / N)$ inside the period interval $-\pi<\phi \leq \pi$ and continued periodically outside it [20, 21].3 Note the scaling of $j$ with the ring length $L=N a$ [20, 21], so that the current vanishes in the bulk limit $L \rightarrow \infty$, as expected.

We now consider the situation where in an otherwise perfect ring there is a single link where the hopping rate is $t_{\mathrm{hop}} \rho$, f rather than $t_{\mathrm{hop}}$, see Figure 1. By rotational symmetry, we thus have a defect boundary condition $\psi(N+1)=\kappa \psi(1)$ with a complex defect strength $\kappa=\rho e^{-i \phi}$. Formally, the diagonalization of the free fermion $X X$ Hamiltonian (4) proceeds along exactly the same lines as had been developed earlier for the treatment of the

\footnotetext{
${ }^{3}$ To facilitate comparison between the models considered here, signs and phases are arranged such as to reproduce this result for the defectless case throughout.

${ }^{4}$ The impurities studied here are only forward scattering.
} 
quantum Ising chain in the presence of point defects (or, equivalently, the classical 2D Ising model with line defects) [1, 22, 23]. Through a JordanWigner transformation and a subsequent canonical transformation, $H$ can be written in terms of free fermionic harmonic oscillators $\eta_{k}$ [24]

$$
H=\sum_{k} \Lambda_{k}\left(\eta_{k}^{\dagger} \eta_{k}-\frac{1}{2}\right)
$$

where $\Lambda_{k}=-t_{\text {hop }} \cos k$ and the values of $k$ are determined by solving

$$
\rho^{2} \sin (k(N-1))+2 \rho \cos \phi \sin k-\sin (k(N+1))=0
$$

The physical ground state is obtained by filling up the lowest $M=N / 2$ one-fermion states. Formally, the ground state energy $-\frac{1}{2} \sum_{k} \Lambda_{k}$ can now be calculated and the current $j$ be obtained. Practically, this is not straightforward, since there is no closed solution to (7) for $|\rho| \neq 1$.

However, to a collective effect as a persistent current only the lowest excited levels should make a significant contribution. Plotting $\Lambda_{k}$ as a function of $k$, one sees that for half-filling, the lowest modes occur at values of $k$ around $\frac{\pi}{2}$ and $\frac{3 \pi}{2}$. Linearizing $\Lambda_{k}$ close to the Fermi energy, we can therefore replace the exact $H$ by an effective low-energy Hamiltonian $H_{\text {eff }}$ of the form $H_{\mathrm{eff}}=\frac{2 \pi t_{\mathrm{hop}}}{N} \sum_{r=0}^{\infty}\left[\left(r+\frac{1}{2}-\mathcal{D}\right) n_{r}^{(-)}+\left(r+\frac{1}{2}+\mathcal{D}\right) n_{r}^{(+)}\right]-\frac{t_{\mathrm{hop}} \pi c_{\mathrm{eff}}}{6 N}+\mathcal{O}\left(N^{-2}\right)$

where $n_{r}^{( \pm)}$are fermionic number operators representing the two lowest modes and where the one-fermion energy shift is $\mathcal{D}=\mathcal{D}(\rho, \phi)$, such that $\mathcal{D}(1,0)=0$. The same form had been found before for the Ising quantum chain with defects 22, 23]. 
In (8) we have dropped a constant contribution of the form $F N+S$. However, these terms have no bearing on the persistent current. To see this we use the analogy of the $X X$ chain (田) with the Ising quantum chain. In turn, the Ising quantum chain can be viewed as the Hamiltonian limit of a two-dimensional classical Ising system, making contact with standard thermodynamics. From this, we recognize $F$ as the bulk free energy density per site and $S$ corresponds to a surface free energy term. This interpretation is carried over to the $X X$ chain. Since the bulk free energy density $F$ is independent of any boundary condition, it is thus also independent on the complex defect strength $\kappa$. On the other hand, in the same framework the surface free energy $S$ may be sensitive to boundary effects. Because of the gauge transformation (ㄱ) , the flux $\phi$ can also be viewed as a bulk interaction, and it follows that $S$ will merely depend on the real defect strength $\rho$, but not on $\phi$. In conclusion, since $F, S$ are both independent of $\phi$, eq. (8) contains all the information required for the determination of the persistent current through (5).

Because the dispersion relation close to the Fermi energies is linear, the effective Hamiltonian represents a conformally invariant system [2, 3, 4]. Conformal invariance allows to relate the (half-filled) physical ground state energy

$$
E_{0}=\sum_{-\frac{\pi}{2}<k \leq \frac{\pi}{2}} \Lambda_{k}=2 E_{0, \mathrm{eff}}
$$

to the constant $c_{\text {eff }}$ and furthermore to the one-fermion energy shift $\mathcal{D}$. Here, $E_{0, \text { eff }}$ is the ground state energy of $H_{\text {eff }}$ and the factor 2 comes from the symmetry of $\Lambda_{k}$ at half-filling.

One of the main results of $2 D$ conformal invariance (e.g. [2, 2, 团), applied 
to critical classical spin sytems, states that it acts as a dynamical symmetry for the associated quantum chain of $N$ sites, with Hamiltonian

$$
H=\frac{2 \pi}{N}\left(L_{0}+\bar{L}_{0}\right)-\frac{\pi c}{6 N}
$$

where $L_{0}, \bar{L}_{0}$ are generators of the Virasoro algebra and $c$ is the central charge. Here, the quantum Hamiltonian $H$ is obtained from the transfer matrix $\mathcal{T}=\exp (-\tau H)$ of the classical $2 D$ spin model, see [1, 3, \#. As it stands, (10) applies to systems without any defects at all and in particular to quantum chains with periodic boundary conditions. One may easily recover the spectrum-generating conformal algebra for the excitations described by $H_{\text {eff }}$ above the Fermi threshold, in complete analogy with the familiar construction for critical Ising spin systems, see [3, 4].

Now, introducing non-periodic boundary conditions (which may also be viewed as a point defect in an otherwise periodic quantum chain), it can be shown that the transformations generated by the diagonal subalgebra $K_{n}=L_{n}+\bar{L}_{n}$ are preserved while the other generators $L_{n}-\bar{L}_{n}$ no longer correspond to a symmetry of the system. Such a point defect in the quantum chain Hamiltonian corresponds, via a conformal transformation, to a semiinfinite line defect in the associated $2 D$ spin model, see [1, 4] and references therein. Specifically, for the Ising quantum chain it turns out that the perfect generators $L_{n}$ are modified into Virasoro generators $L_{n}(\mathcal{D})$ depending explicitly on the one-fermion shifts such that [22]

$$
K_{n}=K_{n}(\mathcal{D})=L_{n}(\mathcal{D})+\bar{L}_{n}(\mathcal{D})+\frac{1}{2} \mathcal{D}^{2} \delta_{n, 0}
$$


satisfy a Virasoro algebra with central charge $c=1$ and

$$
H_{\text {eff }}=\frac{2 \pi}{N}\left(K_{0}(\mathcal{D})-\frac{1}{24}\right)
$$

which generalizes (10). Although invariance under the full conformal algebra generated by the set $\left\{L_{n}, \bar{L}_{n}\right\}$ is broken, the diagonal conformal subalgebra generated by the $K_{n}$ still acts as a dynamical symmetry. Thus, $c_{\text {eff }}=\frac{1}{2}-6 \mathcal{D}^{2}$ [22] and the only change in the ground state energy of $H$ which can give rise to a persistent current is

$$
\delta E_{0}=2 \delta E_{0, \mathrm{eff}}=(2 \pi / N) t_{\mathrm{hop}} \mathcal{D}^{2}
$$

This is the desired result, which relates the current $j$ to the shift $\mathcal{D}$ of the lowest excitations.

To calculate $\mathcal{D}$ to leading order in $1 / N$, we first shift $k$ to $k+\pi / 2$ in order to have $k=0$ at the Fermi surface and then let $k=\alpha / N$ in (7). This gives $\cos \alpha=(-1)^{M}\left[2 \rho /\left(1+\rho^{2}\right)\right] \cos \phi$. From (8), we also have $k \simeq$ $(2 \pi / N)(\ell+1 / 2 \pm \mathcal{D})$, with $\ell=0,1,2, \ldots$ Using these two formulas for $k$, we find $\mathcal{D}=\mathcal{D}(\rho, \phi)$. From (5) the current $j=-\partial\left(\delta E_{0}\right) / \partial \phi$ is obtained as a function of the phase $\phi$. Explicitly,

$$
j(\phi)=t_{\text {hop }} \frac{u \arccos (u \cos \phi) \sin \phi}{\pi \sqrt{1-u^{2} \cos ^{2} \phi}} \frac{1}{N}, u=\frac{2 \rho}{1+\rho^{2}}
$$

and we find again the scaling $j \sim N^{-1}$. Note that this expression is invariant under the transformation $\rho \rightarrow 1 / \rho$ which reflects that the defect, even for $\rho>1$, disturbs the coherence of the many-particle system. As it stands, (14) is correct for $M$ odd. For $M$ even, merely replace $\phi \rightarrow \phi-\pi$. The sizeindependent current amplitude $j(\phi) N$ is shown in Figure 2 a for an entire 


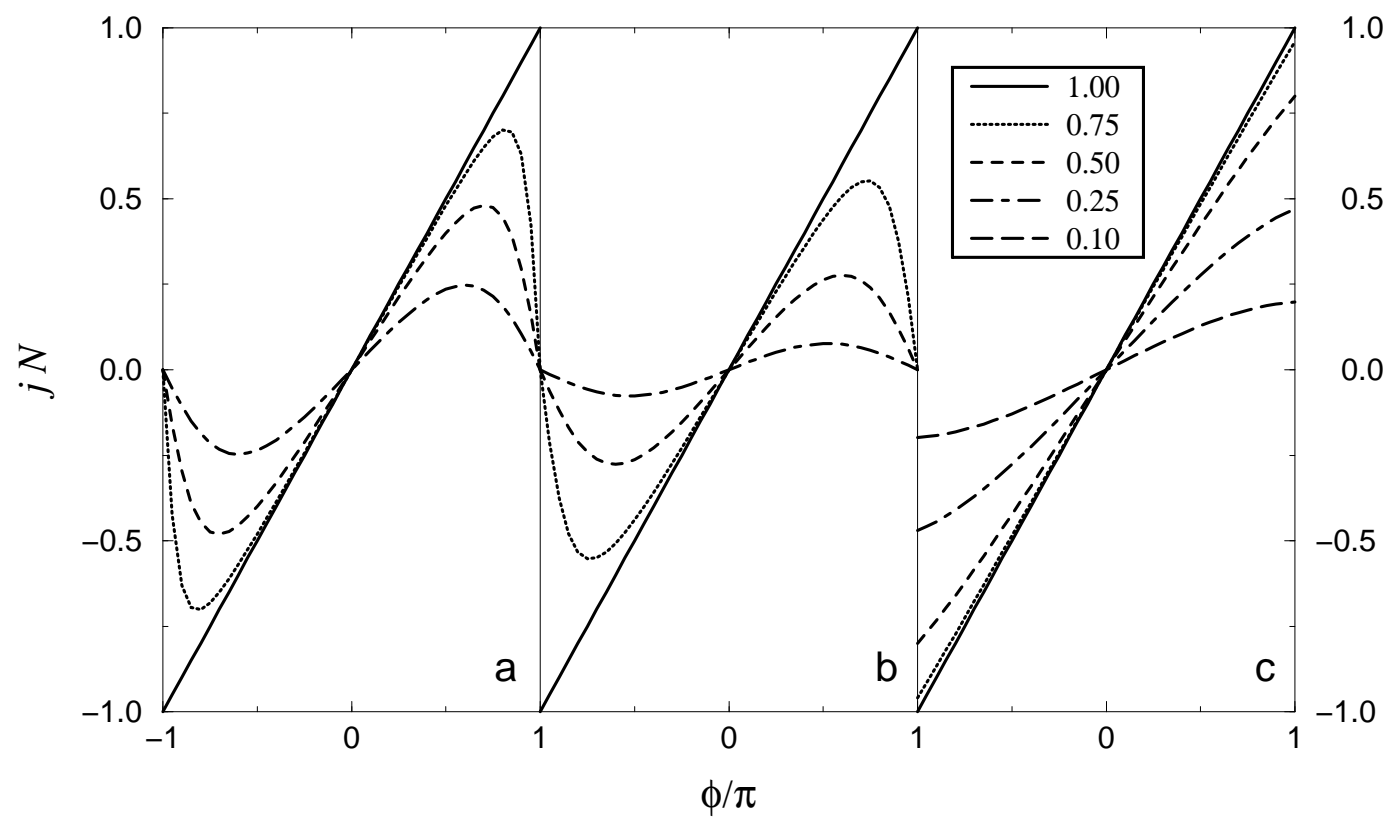

Figure 2: Persistent current amplitude $j(\phi) N$ in the tight-binding model for (a) a single defect with defect strength $\kappa=\rho e^{-i \phi}$ for $M=N / 2$ odd. For two opposite defects with strengths $\kappa_{1}=\rho e^{-i \phi}$ and $\kappa_{2}=\rho$ the persistent current is shown for (b) $N / 2$ even and (c) $N / 2$ odd. The curves correspond to different values of $\rho$ as indicated and $t_{\text {hop }}=1$. 
period of the phase $\phi$ and for several values of the defect coupling $\rho$. While the periodicity with $\phi$ is kept unchanged with respect to the perfect case, the amplitude decreases rapidly with increasing values of $|\rho-1|$. Of course, this result can be checked through direct numerical diagonalization of the tight-binding Hamiltonian (4) and may also be reproduced from a simple continuum description of the model [12].

For two defects which are placed opposite to each other (Figure 11), we find in an analogous fashion

$$
\begin{aligned}
j_{ \pm}(\phi) & =t_{\mathrm{hop}} \frac{a \arccos (a \cos \phi \pm b) \sin \phi}{\pi \sqrt{1-(a \cos \phi \pm b)^{2}}} \frac{1}{N} \\
a & =\frac{4 \rho_{1} \rho_{2}}{\left(1+\rho_{1}^{2}\right)\left(1+\rho_{2}^{2}\right)}, \quad b=\frac{\left(1-\rho_{1}^{2}\right)\left(1-\rho_{2}^{2}\right)}{\left(1+\rho_{1}^{2}\right)\left(1+\rho_{2}^{2}\right)}
\end{aligned}
$$

where + corresponds to $N / 2$ even, - corresponds to $N / 2$ odd and $\rho_{1,2}$ are the couplings at the defects. For $\rho_{1}=1$ or $\rho_{2}=1$, one simply recovers (14). In Figure 2 bc, we show the current amplitude for the number of particles $M=N / 2$ even and odd, respectively and for $\rho_{1}=\rho_{2}=\rho$. In contrast to the single-defect case, there is a clear parity effect. For an even number of particles, the amplitude is broadly similar to the single-defect case (up to a renormalization of $\rho$ ). In particular, the current $j(\phi)$ is a continuous function of $\phi$ over the entire period. For an odd number of particles, the flux-dependence of the current is almost linear for a wide range of values of $\rho$ and there is a jump in $j$ at the ends of the period interval. Also, the presence of the defects merely changes the prefactor.

We now consider the case of two defects placed anywhere on the ring. The first one may always considered to be placed at the origin, while the 
second one will sit at the site $R=\lambda N$. The continuum dispersion relation remains unchanged, but (可) has to be replaced by

$$
\left(1-\rho_{1}^{2}\right)\left(1-\rho_{2}^{2}\right) \cos [\alpha(1-2 \lambda)] \pm\left(1+\rho_{1}^{2}\right)\left(1+\rho_{2}^{2}\right) \cos \alpha \pm 4 \rho_{1} \rho_{2}(-1)^{M} \cos \phi=0
$$

where $N=2 M$ is the number of sites, $k=\alpha / N$ (after the shift $k \rightarrow k+\pi / 2$ ) and $+(-)$ corresponds to $R$ even (odd). Since $\lambda=R / N$ is rational, we may write $\lambda=p / q$ with $p, q$ mutually prime. Eq. (16) then becomes a polynomial equation of degree $\gamma(q)$ in the variable $\cos (2 \alpha / q)=\cos \left(2 \pi \mathcal{D}^{(i)}\right)$, where $\gamma(q)=q / 2$ if $q$ is even and $\gamma(q)=q$ if $q$ is odd. The current is then written in terms of the $\gamma(q)$ distinct one-fermion shifts as

$$
j=-\frac{\partial E_{0}}{\partial \phi}=-\frac{4 \pi \gamma(q)}{N} \sum_{i=1}^{\gamma(q)} \mathcal{D}^{(i)} \frac{\partial \mathcal{D}^{(i)}}{\partial \phi}
$$

It is straightforward to extend the treatment to an arbitrary number of point defects, following the lines of [23] for the Ising quantum chain.

These results were derived by going back to the explicit diagonalization of the underlying tight-binding model and using the analogy with the quantum Ising chain for defect boundary conditions. In the Ising quantum chain, a defect boundary condition corresponds to a marginal perturbation [1] which leads to a shift $\mathcal{D}$ in the one-fermion energies which depends continuously on the defect strengths and consequently to a continuously varying effective central charge $c_{\text {eff }}$. Extending existing field-theoretical treatments of defect lines in $2 D$ models [25, 26, 27] to complex defect strengths might provide further insight into the problem at hand.

\footnotetext{
${ }^{5}$ For example, the generators (11) can also be obtained using the automorphisms of the underlying Kac-Moody algebra [28]. This more abstract construction is independent of a free-fermion realization of the model.
} 
Having seen that at least in the tight-binding model with point defects, the persistent currents are related to the shift in the low-lying excitations above the Fermi energy, we now briefly discuss persistent currents in aperiodically modulated rings threaded by a magnetic flux. The Hamiltonian is

$$
H=-\sum_{n=1}^{N} t_{n}\left[\psi^{\dagger}(n+1) \psi(n)+\psi^{\dagger}(n) \psi(n+1)\right]
$$

at half-filling, together with the flux boundary condition used before and where the aperiodic modulation in the hopping matrix element $t_{n}$ is generated through a binary substitution sequence [29, 30]. We shall consider here

$$
\begin{array}{cc}
\text { Fibonacci : } & A \rightarrow A B, B \rightarrow A \\
\text { Thue-Morse }: & A \rightarrow A B \quad, B \rightarrow B A
\end{array}
$$

and the sequences are obtained by starting from the letter $A$ and iterating the above procedure many times. For example, using the Thue-Morse substitution rules, the first three iterations give the following sequences

$$
\begin{array}{cc} 
& A \\
i=1: & A B \\
i=2: & A B B A \\
i=3: & A B B A B A A B
\end{array}
$$

A modulation in (18) is obtained by letting $t_{n}=t_{\text {hop }}$ if the site $n$ is the letter $A$ and $t_{n}=t_{\text {hop }} \rho$ otherwise. For such aperiodic modulation the cumulated deviation from the average hopping rate $\bar{t}, \Delta(L):=\sum_{k=1}^{L}\left(t_{k}-\bar{t}\right)$ scales with the chain length $L$ as $\Delta(L) \sim L^{\omega}$, where $\omega$ is the so-called wandering exponent, caracteristic of the sequence [29, 30]. If $\omega<0$, as it is the case for the two sequences under consideration in this paper, $\Delta(L)$ remains bounded for $L \rightarrow \infty$. 


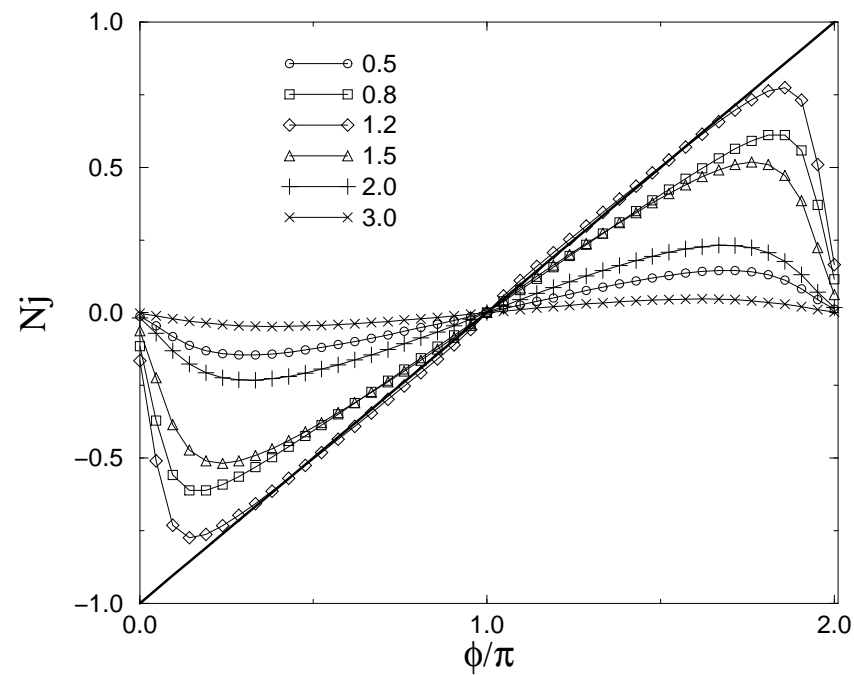

Figure 3: Current amplitude $N j$ for a ring modulated with the Fibonacci sequence, $N=144, t_{\text {hop }}=1$ and different values of $\rho$.

For critical spin systems, Luck 31] has formulated a criterion which for a given spin system and a given sequence predicts whether the aperiodic modulation is relevant, marginal or irrelevant. According to the Luck criterion, the modulations generated by both the Fibonacci and the Thue-Morse sequence are irrelevant for the Ising quantum chain and the low-lying excitations of $H$ are in the $N \rightarrow \infty$ limit not affected by the modulation. Because of the close connection of the tight-binding model with the critical point of the Ising quantum chain, we expect to retain the scaling $j \sim N^{-1}$ of the persistent current, but we are curious about the flux-dependence of $j(\phi)$.

In Figure 3, we show the current amplitude $j N$ as obtained for several values of $\rho$ for an aperiodic ring modulated with the Fibonacci sequence. 


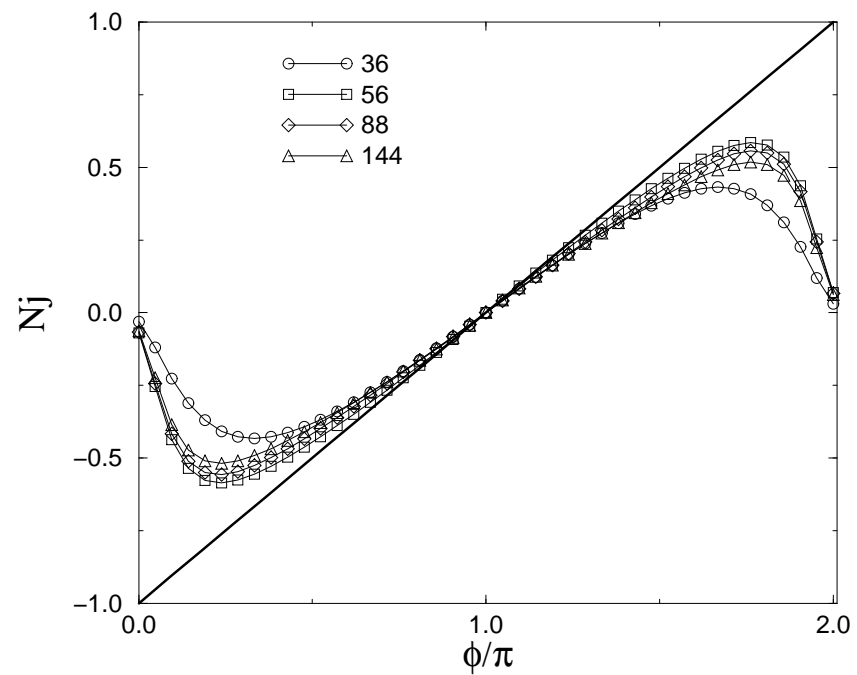

Figure 4: Current amplitude $N j$ for a ring modulated with the Fibonacci sequence, $\rho=1.5$ and varying number of sites $N$.

We also include the straight line behaviour for the unmodulated ring for comparison. We observe that the modulation reduces the current density with respect to the unmodulated case and that the dependence of $j(\phi)$ on the flux is similar to the one found for the single defect in Figure 2a. In Figure 4 , we illustrate for fixed $\rho$ the convergence of the finite-size data with $N$. We see that quite large lattices are needed to achieve convergence of the current amplitude. Finally, in Figure 5 the current amplitudes for the Thue-Morse sequence are displayed. In this case, the convergence with $N$ is much faster than for the Fibonacci sequence and already for $N=64$, there is no perceptible change of the amplitude with $N$.

Remarkably, the current depends on the flux in very different ways for the 


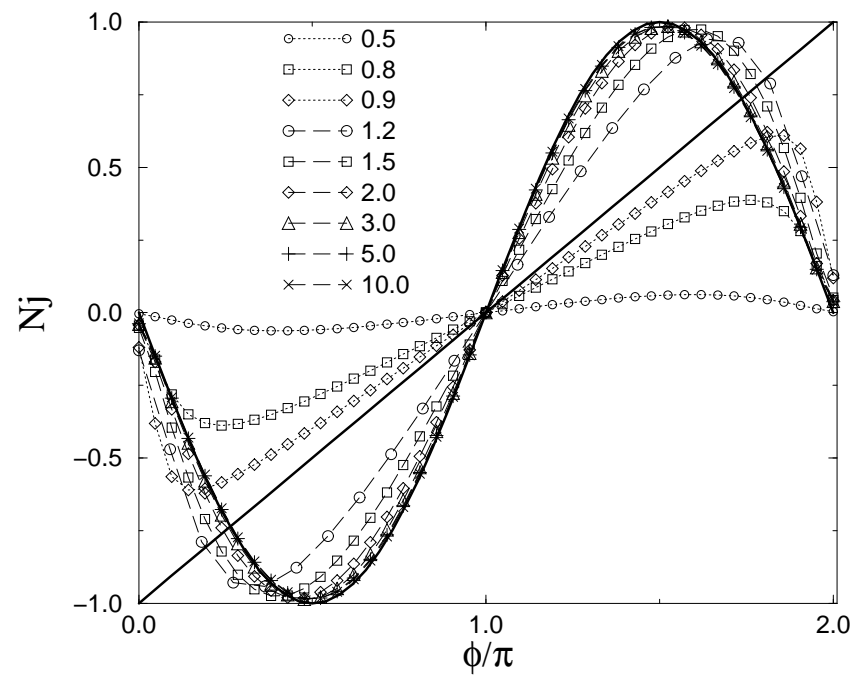

Figure 5: Current amplitude $N j$ for a ring modulated with the Thue-Morse sequence, $N=64, t_{\text {hop }}=1$ and several values of $\rho$.

two sequences, although we observed indeed the expected scaling $j \sim N^{-1}$. For the Fibonacci sequence, the amplitude $N j(\phi)$ with $\rho \neq 1$ is always smaller than the amplitude for the non-modulated ring $\rho=1$, independently of whether $\rho$ is larger or smaller than unity. That is not so for the Thue-Morse sequence. Rather, the amplitude $N j$ is weakened for $\rho<1$, but for $\rho>1$, the amplitude becomes considerably larger than in the unmodulated case. In fact, for $\rho \rightarrow \infty$, we recognize a limit form $N j(\phi)=-\sin \phi$. In spite of being irrelevant to the critical behaviour of Ising quantum chains, the details of the aperiodic modulation are important for the flux-dependence of the persistent current. These finer properties are not reflected in the low-lying excitation spectrum of the Hamiltonian. 
In conclusion, the role of defects on the persistent current in mesoscopic rings was studied through a simple tight-binding model. From the analogy with the local critical behaviour of conformally invariant spin systems with defect lines, when extended to complex defect strengths, explicit formulae for the persistent current were obtained. The flux-dependence of the current shows an unexpected parity effect. In addition, the persistent current was found for two types of aperiodically modulated rings. The flux-dependence of the current $j(\phi)$ cannot be obtained from a consideration of the low-lying excitation spectrum alone, in contrast to the case of point defects. It would be interesting to see to what extent these observations can be generalized beyond the simple tight-binding model.

Acknowledgement: It is a pleasure to thank H. Schoeller for very useful discussions.

\section{References}

[1] F. Iglói, I. Peschel and L. Turban, Adv. Phys. 42, 683 (1993)

[2] J.L. Cardy, Scaling and Renormalization in Statistical Physics, Cambridge University Press (Cambridge 1996)

[3] P. di Francesco, P. Mathieu and D. Sénéchal, Conformal Field Theory, Springer (Heidelberg 1997)

[4] M. Henkel, Conformal Invariance and Critical Phenomena, Springer (Heidelberg 1998), Chap 15

[5] V. Chadrasekhar, R.A. Webb, M.J. Brady, M.B. Ketchen, W.J. Gallagher and A. Kleinsasser, Phys. Rev. Lett. 67, 3578 (1991)

[6] D. Mailly, C. Chapelier and A. Benoit, Phys. Rev. Lett. 70, 2020 (1993) 
[7] I.O. Kulik, JETP Lett. 11, 275 (1970)

[8] M. Büttiker, Y. Imry and R. Landauer, Phys. Lett. 96A, 365 (1983)

[9] H.F. Cheung and E.K. Riedel, Phys. Rev. B40, 9498 (1989)

[10] A.A. Zvyagin, Sov. Phys. Solid State, 32, 905 (1990)

[11] B.S. Shastry and B. Sutherland, Phys. Rev. Lett. 65, 243 (1990)

[12] A.O. Gogolin, Phys. Rev. Lett. 71, 2995 (1993); A.O. Gogolin and N.V. Prokofev, Phys. Rev. B50, 4921 (1994)

[13] I.V. Krive, P. Sandström, R.I. Shekhter and M. Jonson, Phys. Rev. B54, 10342 (1996); P. Sandstöm and I.V. Krive, Ann. of Phys. 257, 18 (1997)

[14] G. Bouzerar, D. Poilblanc and G. Montamboux, Phys. Rev. B49, 8258 (1994); B52, 10772 (1995); F. Mila and D. Poilblanc, Phys. Rev. Lett. 76, 287 (1996)

[15] M. Abraham and R. Berkovits, Phys. Rev. Lett. 70, 1509 (1993)

[16] E.S. Sørensen, S. Eggert and I. Affleck, J. Phys. A26, 6757 (1993); S.J. Qin, M.Fabrizio, L. Yu, M. Oshikawa and I. Affleck, Phys. Rev. B56, 9766 (1997)

[17] A. Völker and P. Kopietz, Z. Phys. B102, 545 (1997); Mod. Phys. Lett. B10, 1397 (1997)

[18] S.J. Qin, M. Fabrizio and L. Yu, Phys. Rev. B54, R9643 (1996)

[19] D. Loss, Phys. Rev. Lett. 69, 343 (1992)

[20] A.A. Zvyagin and I.V. Krive, Low Temp. Phys. 21, 533 (1995)

[21] Y. Imry, Introduction to Mesoscopic Physics, Oxford University Press (Oxford 1997)

[22] M. Henkel and A. Patkós, Nucl. Phys. B285, 29 (1987)

[23] M. Henkel, A. Patkós and M. Schlottmann, Nucl. Phys. B314, 609 (1989)

[24] One can find the explicit derivation in the classical paper E. Lieb, T. Schultz and D. Mattis, Ann. of Phys. 16, 407 (1961)

[25] G. Delfino, G. Mussardo and P. Simonetti, Nucl. Phys. B432, 518 (1994)

[26] R. Konik and A. LeClair, preprint hep-th/9703085 
[27] M. Oshikawa and I. Affleck, Nucl. Phys. B495, 533 (1997)

[28] M. Baake, P. Chaselon and M. Schlottmann, Nucl. Phys. B314, 625 (1989)

[29] M. Quéffelec, in A. Dold and B. Eckmann (Eds) Substitution Dynamical Systems - Spectral Analysis, Lecture Notes in Math., vol. 1294, Springer (Heidelberg 1987), p. 97

[30] J.M. Dumont, in J.M.Luck, P. Moussa and M. Waldschidt (eds) Number Theory and Physics, Springer (Heidelberg 1990), p. 185

[31] J.M. Luck, J. Stat. Phys. 72, 417 (1993); Europhys. Lett. 24, 359 (1993) 